\title{
Associate-Image Filtering Method with Enhanced De-noising Feature for Road Detection in Disaster Management
}

\author{
K.Kiran Reddy, P.Bhsskara Redd and P.Amarender Reddy \\ MLRMLR Institute of Technology, Dundigal, Hyd, Telangana, India \\ kiran_kolanu@yahoo.co.in; pbhaskarareddy@rediffmail.com; amarpanyala88@gmail.com
}

\begin{abstract}
Due to rapid urban development, the Geographic Information System (GIS) database needs to be updated with timely and accurate road network information. This paper presents an approach to design a module for image pre-treatment of roads (or roads seeds) and help to decide the most suitable emergency transportation route in disastrous area. Also, in such situation, the quality of the image may degrade during capture or transmission as the entire process becomes prone to noise and instability. Therefore for any kind of information processing or decision making image pre-treatment is a significant part. This paper presents a Multistage Hybrid Median filtering (MHMF) technique to significantly improve noise reduction performance of satellite/aerial road images while preserving the integrity of edge and detail information. Further, the images are divided into subparts and they are processed using the proposed MHMF. Then the two filtered sub-images are combined and we can improve overall performance even further. To support the above claim, a case study has been carried out on two recent natural disasters happened in India along with other benchmark problems and the studies show the effectiveness of the proposed system in real environment.
\end{abstract}

General Terms: Image Denoising

Keywords: Image Preprocessing, Sub-image, Median Filter, Road Detection, GIS, Objective Image Quality Metrics, Signal to Noise Ratio, Image Quality Index.

\section{Introduction}

Road is a major component of lifeline and a vital tool for the transportation of goods and services between different regions. A natural disaster (e.g. earthquake) may severely violate the functioning of roads, and as a result may suspend the emergency response in affected region. Therefore it is extremely necessary to collect the post disaster road condition and find out alternate route to establish communication with the zone of interest. However, the entire picture of damaged condition in large extent cannot be attained in a short time through traditional ways, and in most of the cases it is impossible.

Remote sensing technology with the advantage of rapid collection mechanism of comprehensive and objective data is not influenced by destruction and can monitor the disaster continuously [1]. Especially the advent of high-spatial resolution sensors provides a new way to collect the detailed information of roads, so that the remote sensing technology is applied for damage detection and assessment of roads [2]. Using the basic road data from GIS as the prior knowledge, the system extracts the pre-disaster and post-disaster road image and information from post-disaster remotely sensed imageries, and then detects 
the damaged regions and evaluates the intensity of destruction. Satellite Remote Sensing, when colligated with Geographic Information System (GIS) becomes an extremely valuable tool for Natural Disaster Management. The datasets acquired from Earth observation satellites (EOS) can be used by disaster managers during almost all four phases of Disaster Management; namely mitigation, preparedness, response, and recovery [2]. During response and recovery phases one of the key activities performed by the relief agencies, civil protection bodies, and other disaster management organizations is known as Damage Assessment. This assessment can either be performed by conducting a ground survey or by using satellite images and aerial photographs acquired over the earthquake affected areas. Irrespective of the method used for assessment, the most common output of this process is a Damage Assessment Map. The most effective way to produce these maps is to use satellite images as they are quite readily available and can even report damage for inaccessible regions.

Our original research mainly describes and evaluates an image analysis system for the automated identification of intact roads from high resolution aerial/satellite images. The system is to be designed to support rescue teams during the reaction on natural disasters such as flooding, landslides, earthquakes etc. and help to find the alternate route for fast relief transportation. This paper mainly emphasizes on a sub module design of a road detection system that helps to decide the most suitable emergency transportation route in disastrous area. Also as an additional advantage, it may highlight the regions which are mostly affected by the given natural calamities.

In this paper, the entire work is divided into two phases. At the first phase, we propose a Multistage Hybrid Median filter (MHMF) to address the limitation of most median based filter. In subsequent phase, it is shown that by dividing the input image into sub-images, and performing the proposed MHMF on each sub-image individually, the performance can be significantly improved. It will also be shown that by combining two filtered sub-image representations, the overall performance can be improved even further. This proposed filter is a windowed filter of nonlinear class that easily removes impulse noise while preserving the edges. In comparison with basic version of the median filter, proposed hybrid filter has better edge preserving characteristics which is very helpful for road network and road junction detection and extraction and other image processing applications. To support these assumptions our proposed technique will be compared with current standard noise removing techniques. Initially, the image is corrupted with a very difficult salt \& pepper noise on the sub-section of grayscale images taken from our study area, following a standard verification procedure. In order to demonstrate the efficacy of remote sensing technologies for disaster management, three case studies are presented. They are-

Case-I: the datasets related to the Sumatra earthquake struck South Asia on 26 December 2004 as a test data [3]

Case-II: The category 3 tropical cyclone Hudhud, in 2014 Vizag, India [4].

Case-III: Uttarakhand India torrential rain and cloudbursts, in 2013 [4].

The details of the problem description and the corresponding results were provided in the subsequent sections. The aim of these case studies is to concentrate on the detection of intact road networks under the constraints of an effective disaster management. Those parts of the road network, which are not detected by the system, should be regarded as destroyed and not accessible for rescue and evacuation teams anymore. 
K.Kiran Reddy, P.Bhsskara Redd and P.Amarender Reddy; Associate-Image Filtering Method with Enhanced Denoising Feature for Road Detection in Disaster Management. Transactions on Machine Learning and Artificial Intelligence, Volume 4 No 6 December (2016); pp: 50-63

In summary, the overall objective of this paper is as follows

- To design a sub module for Image pre-treatment for roads (or roads seeds) and help to decide the most suitable emergency transportation route in disastrous area. The goal is to develop a complete real time automated solution to the road extraction problem for high resolution aerial/satellite images.

- A novel Multistage Hybrid Median filtering (MHMF) has been proposed for processing section. This is to overcome the limitation of most median based filter.

- Further, the performance can be significantly improved by extracting the information and decision making in hierarchical manner. This can be accomplished by dividing the input image into sub-images, and performing the proposed MHMF on each sub-image individually. Combining two filtered sub-image representations, and improving the overall performance even further.

The rest of this paper is organized as follows: Section 2 presents some related work on filtering techniques used for noise reduction in the literature. Section 3 describes our proposed experimental setup in detail. Section 4 gives a brief description of the datasets used for experimentation. Experimental results are introduced in Section 5. Finally, Section 6 concludes the paper and lists possible improvements and further work.

\section{Related Work}

The goal of de-noising is to remove the noise while preserving the important image information as much as possible [5] [6]. Linear filtering techniques, such as Wiener filter or match filter, have been used for this purpose for many years. But linear filters may result in some problems, such as blurring the sharp edges, destruction of lines and other finer image details etc. Due to these facts, an alternative filtering technique like nonlinear filtering is necessary. Many works [7] [8] have been reported on image de-noising using nonlinear filters.

Many de-noising methods have been proposed over the years, such as the Wiener filter, wavelet thresholding [9] [10], anisotropic filtering [11], bilateral filtering [12]. Among these, wavelet thresholding has been reported to be a highly successful method. In wavelet thresholding, a signal is decomposed into approximated (low-frequency) and detail (high-frequency) sub-bands, and the coefficients in the detail sub-bands are processed via hard or soft thresholding [9], [10] [13], [14]. Donoho [9] gave a general estimation method of threshold, but the best threshold cannot be found by this method. Chang et al. [14] have used predictive models to estimate the threshold.

In recent years, several methods have been proposed for removing impulsive noise. One of most popular filter is the standard median filter (SMF) [15] which is based on replacing every image pixel with the median value computed within the window without considering the status of (noisy/noise-free) pixels. But it has some disadvantages, for example this method mistakenly destroys edges and the image is blurred. Recently, in response to these difficulties, several variations of median filters have been introduced [16] [17] [18].

To enhance the capability of non-linear filters, the switching concept was introduced in [19]. This is used

to determine the pixel noise of impulse detector. Among the variety of switching based filtering 
techniques, adaptive median filter (AMF) [20] has gained the most popularity, but with this method the quality of image restoration is not satisfactory. In [21], an adaptive median filter that adjusts the window size depending on the input is proposed to trade between detail preservation and noise suppression. For signal-dependent noise reduction some adaptive median-type filters, such as adaptive double window modified trimmed mean [22] have been proposed. Other variations of SMF are weighted median filters (WMF) [23], center weight median filter (CWMF) [24], adaptive CWM (ACWM) [25], Progressive Median Filter (PSMF) [26], Tri-state Median Filter (TMF) [27], Decision-based Median Filter (DBMF) [28], Relaxed Median Filter (RMF) [29], Adaptive Length Median Filter (ALMF) [30] and Alpha Trimmed Median Filter (ATMF) [31]. Most of these algorithms provide suitable and good results at smaller percent of noise levels and find difficulty with higher level noises. A variety of adaptive filtering techniques has been proposed which help in enhancing images degraded by noise [32] [33]. In [34], a novel method of hybrid filter for de-noising digital images corrupted by mixed noise has been presented. The proposed design of hybrid filter utilizes the concept of neuro fuzzy network and spatial domain filtering.

Several fuzzy filters for noise reduction have been developed, e.g., the well-known FIRE-filter [35], the weighted fuzzy mean filter [36]. The adaptive weighted fuzzy mean filter [37] (AWFM), the histogram adaptive fuzzy filter [38] (HAF) and the adaptive fuzzy switching filter (AFSF) which uses the maximumminimum exclusive median filter [39], are other examples of the state-of-the-art methods. In [40], Dimitri Van De Ville et.al. presented a new fuzzy filter for the noise reduction of images corrupted with additive noise.

Most of these algorithms provide suitable and good results at smaller percent of noise levels and find difficulty with higher level noises. Moreover, most median based filters use median value or weighted median value from the whole window as the estimated value. However, since the noisy pixels are replaced by median value in their vicinity without taking into account the local features such as the possible presence of edges, details and edges are not recovered satisfactorily [6].

\section{Proposed Method}

In this paper, initially a Multistage Hybrid Median Filter (MHMF) has been proposed for preserving details of images. The algorithm of the proposed technique is given in TABLE 1. It is shown that the new filter can smooth noise more efficiently than the other standard median filters. Let be a discrete two-dimensional sequence, and consider the set of elements inside a pixel. Define the square window $\mathrm{W}$ centered at the following four subsets of the window W Suppose that are the median values of the elements in the four subsets, respectively, and Then the output of the proposed hybrid median filter is defined by this filter always gives at least a fourfold improvement in edge shift over that of the basic median filter. One of the advantages of this MHMF is its ability to preserve details of images because data from different spatial directions are ranked separately.

Encouraged by the good results obtained with this proposed method (see Section 5 for details) we further tried to improve the filtering results. We tried to split the original image in subimages and filter each subimage individually by the proposed approach. First we split the image into two halves vertically (thus obtaining two sub-images of the original image) and then apply the filter to each half individually. In the next phase, we split the image horizontally and again apply the filter to each half individually. Finally the filtered images obtained from both the horizontal and vertical processing sections (see Section 3 for details) are combined into a joint representation. Let be the image split vertically and each half filtered 
K.Kiran Reddy, P.Bhsskara Redd and P.Amarender Reddy; Associate-Image Filtering Method with Enhanced Denoising Feature for Road Detection in Disaster Management. Transactions on Machine Learning and Artificial Intelligence, Volume 4 No 6 December (2016); pp: 50-63

with multistage hybrid filter individually, and let be the same for horizontally split images and let be our proposed modification. Then we can represent the final image as given by

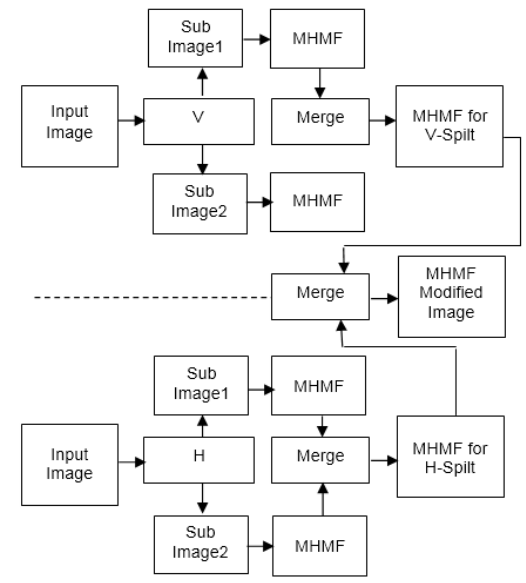

Fig. 1 Multistage Sub-Image Filtering Technique

We have shown that by combining two filtered sub-image representations, we can improve overall performance even further. Since IMHMV and IMHMH scored fair with very high reliability in our tests, we decided to keep the whole IMHMV and IMHMH (chosen based on experimental results), to higher its influence on the final representation. This combination produced highest results in our experiments and was kept as a final representation. The whole procedure is summarized in Fig. 1, and examples of the filtered image can be seen in Fig. 2, and Fig. 4-13 for one sample test input images from our database. We will show in the following section that our method yields superior results, and therefore justifies further research of the hybrid median filtering variations as a means of simple yet efficient image pre-processing technique.

TABLE 1: Proposed Multistage Filtering Algorithm

\begin{tabular}{|c|l|}
\hline Step1 & $\begin{array}{l}\text { Consider be a discrete two-dimensional image, and consider the set of elements inside } \\
\text { a square window W centered at the pixel and define the four subsets of the window } \\
\text { W, as given in equation (1) to (4). }\end{array}$ \\
\hline Step 2 & Compute the median values of the elements in the four subsets, respectively. \\
\hline Step 3 & Compute min and max value of as given in as given in equation (5) and (6). \\
\hline Step 4 & The output of the proposed hybrid median filter is defined by \\
\hline Step5 & $\begin{array}{l}\text { Compute: Split the input image into two halves vertically (thus obtaining two sub- } \\
\text { images of the original image) and then apply the filter to each half individually. }\end{array}$ \\
\hline Step6 & $\begin{array}{l}\text { Compute: split the image horizontally and again apply the filter to each half } \\
\text { individually. }\end{array}$ \\
\hline Step 7 & \begin{tabular}{l} 
Compute the resultant image by as per equation (8) \\
\hline
\end{tabular}
\end{tabular}

\section{Data Sets for Experiments}

The experimental data sets for this work consist of two pairs of satellite/aerial imagery captured on different dates. Three test areas are chosen for this procedure and details of the data sets are given below. 
CASE STUDY-I: The first study is on the datasets related to the Sumatra earthquake in South Asia on 26 December 2004 [3]. The FORMOSAT-2 satellite successfully acquired several post-tsunami images of the hazardous areas, both Puhket, Thailand and Banda Aceh, Indonesia on 28 December.

CASE STUDY-II: On 12 October 2014, the category 3 tropical cyclone =Hudhud' made landfall on the coast of Andhra Pradesh, near the city of Visakhapatnam (also known as Vizag), India.

CASE STUDY-III: The Himalayan states of Himachal Pradesh and Uttarakhand were hit by torrential rain and cloudbursts in certain locations from 14th June 2013 to 17th June 2013, which triggered landslides and flash floods in multiple locations in these states.

For case study-II and III, 25 images were captured respectively for each case. For creating the data set, selected sections ( $512 \times 512$ pixels) of scenes from satellite images were considered from acquired images from internet [4].

\section{Experiments and Results}

In this section, experimental results are reported to validate the proposed method. To test our approach, we have used the satellite/aerial images of the three cities of Vizag, Uttarakhand and Puhket, Thailand, Indonesia as a case study. Our approach is implemented with Matlab and tested on a Core i5 $2.67 \mathrm{GHz}$ PC with 4GB RAM. Simulations were carried out in MATLAB. To evaluate the denoising and enhancement of image objectively [41], following image metrices were considered: Mean Square Error ( ), Root Mean Square Error (RMSE) [41], Signal to Noise Ratio (SNR) [41], Peak Signal to Noise Ratio (PSNR) [41], Normalized Cross-Correlation (NCC) [41] [6], Average Difference (AD) [41], Structural Content (SC) [41], Maximum Difference (MD) [41], Mean Absolute Error (MAE) [41], Normalized Absolute Error (NAE) [41], Image Quality Index (Q) [42] and Enhancement Measurement Error (EME) [48]. Higher and mean more powerful denoising capacity of the algorithm. Lower means nearer distance of the original image and the denoised image.

Test images from the database have been experimented, and each image has $4 \%$ (i.e., $p=0: 0.04$ ) and 20\% (i.e., $p=0: 0.2$ ) of total pixels being corrupted by impulse noise which is uniformly distributed over the range of $[0 ; 255]$. The image shown here is from the affected areas of Vizag costal area. Due to space constraint all the results were reported only with respect to a single image from case study II. However extensive study has been performed with all the images from other cases also. The objective measures for different filters are illustrated in Table 2 and Table 3 for one sample test input images of the study area Vizag city costal region respectively under different noise conditions. The performance metrics show that amongst the different type of noise reduction filters listed in Table $2 \& 3$, the proposed method removes substantial amount of noise and also preserves edges and details.

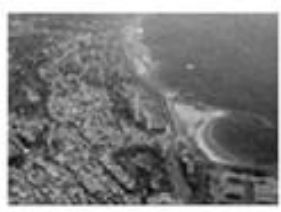

(a)

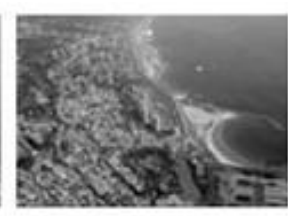

(b)

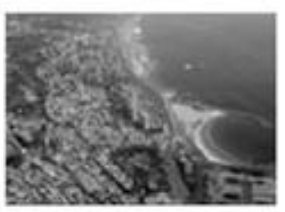

(c)

Fig 2: Case-II-Vizag city costal region: (a) Original Image corrupted by noise density 0.04 (b) Standard Median Filtered Image (c) Proposed Hybrid Multistage Filter 
K.Kiran Reddy, P.Bhsskara Redd and P.Amarender Reddy; Associate-Image Filtering Method with Enhanced Denoising Feature for Road Detection in Disaster Management. Transactions on Machine Learning and Artificial Intelligence, Volume 4 No 6 December (2016); pp: 50-63

MHMF vertical (MHMV): Our proposed MHM filtering of two subimages obtained by vertically dividing the input image into two halves prior to filtering and then filtering each of them. The resulting image is obtained by concatenating the two filtered halves (Fig. 6-7(a), Fig. 8-9).

MHMF horizontal (MHMH): The same procedure as in MHMV with the exception of an image being horizontally divided (Fig. 4-5, Fig. 10-11(a)).

MHMF modified (MHMFMOD): Method proposed in Section 3, consisting in combining results from MHMV and MHMH (Fig. 7(b) and 11(b)).

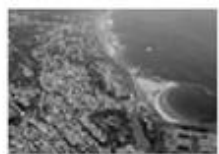

(a)

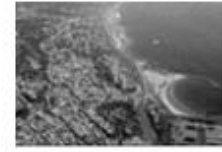

(d)

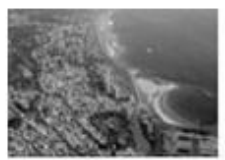

(g)

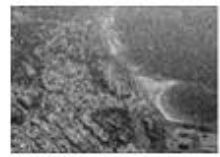

(j)

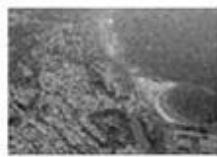

(b)

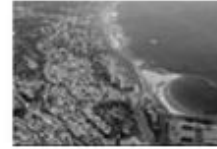

(e)

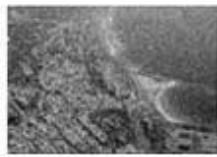

(h)

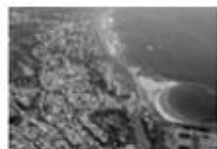

(k)

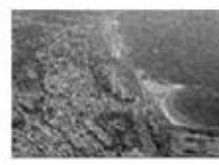

(c)

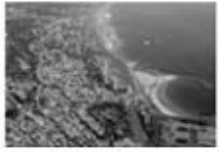

(f)

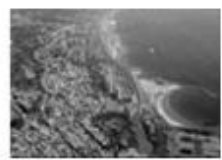

(i)

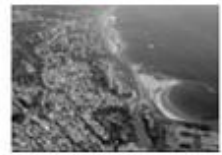

(I)

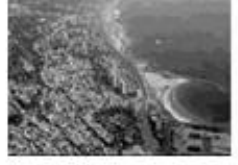

(m)

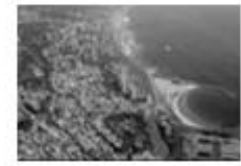

(p)

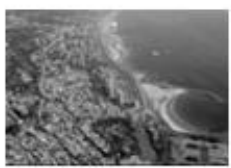

(s)

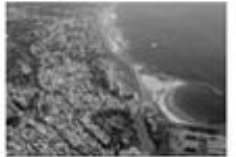

(n)

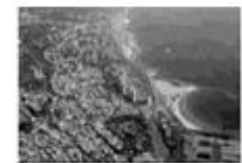

(a)

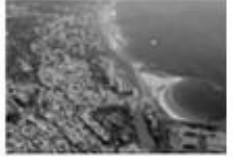

(o)

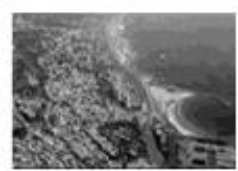

(r)

Fig 3: Case-II-Vizag city costal region: a) Original Aerial Road Image b) Image corrupted by Salt and Pepper noise with density 0.04 c) Average Filtered image d) MF e) HMF f) AMF image g) PSMF image h) Adaptive local filtered image i) RMF j) Wiener filtered image $k$ ) WMF image I) CWMF $m$ ) TMF n) DBA o) Bilateral Filtering $p$ )

Proposed Filter q) MHMV Filtered Image r) MHMH Filtered Image s) MHMFMOD Image

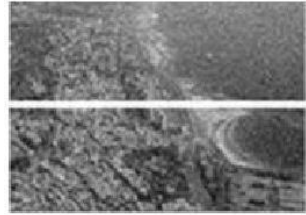

(a)

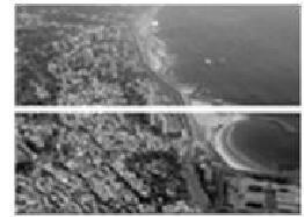

(b)

Fig 4: Case-II-Vizag city costal region: (a) Two sub-images of the original Aerial image after dividing the input image horizontally. Each subimage corrupted by noise density 0.2, (b) Two Horizontal Sub-Images Filtered separately (MHMH) by Our Proposed MHM Filter (MHMF) 


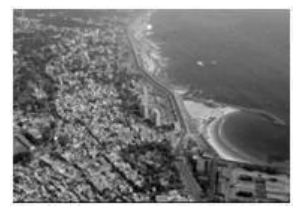

(a)

Fig 5: Case-II-Vizag city costal region: Proposed HMM Filtered Merged Image after performing Horizontal Sub-Image filtering in Fig. 4.

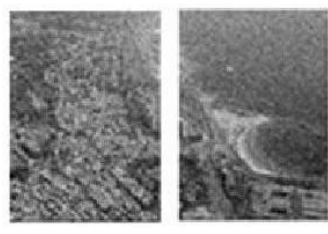

(a)

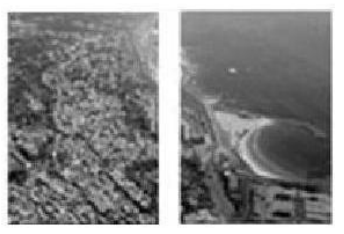

(b)

Fig 6: Case-II-Vizag city costal region: (a) Two sub-images of the original image after dividing the input image vertically. Each subimage corrupted by noise density 0.2 (b) Two vertical Sub-Images Filtered separately (MHMV)

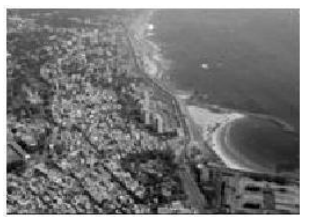

(a)

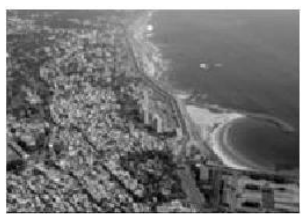

(b)

Fig 7: (a) Case-II-Vizag city costal region: Proposed Hybrid Multistage Median Filtered Merged Image after performing vertical Sub-Image filtering in Fig. 6 (b) Case-II-Vizag city costal region: Proposed Combination (MHMFMOD)

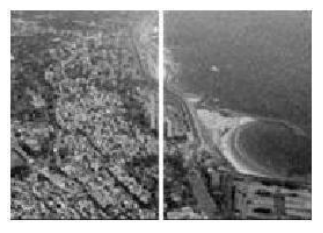

(a)

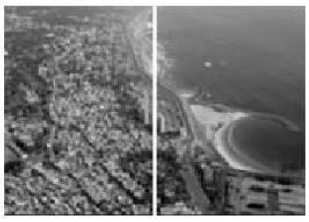

(b)

Fig 8: Case-II-Vizag city costal region: (a) Two Sub-images of the original image by dividing the input image vertically. Each subimage corrupted by noise density 0.04 (b) Two Vertical Subimages Filtered Separately (MHMV) by Proposed Filter (MHMF)

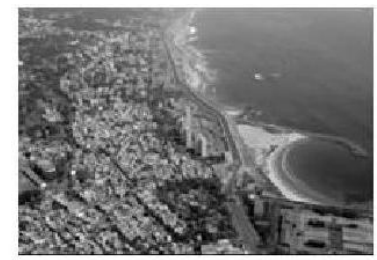

(a)

Fig 9: Case-II-Vizag city costal region: Proposed Multistage Hybrid Median Filtered Merged Image after Performing Vertical Sub-Image filtering (MHMV) 


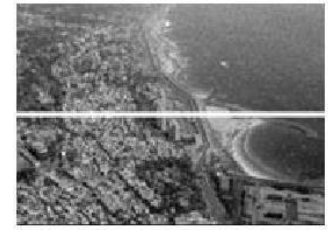

(a)

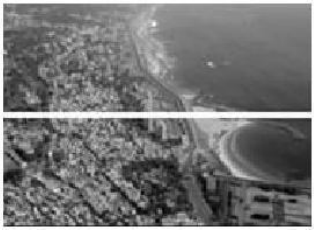

(b)

Fig 10: Case-Il-Vizag city costal region: (a) Two Sub images of the original image by dividing the input image horizontally. Each sub image corrupted by noise density 0.04 (b) Two Horizontal Sub-Images Filtered separately

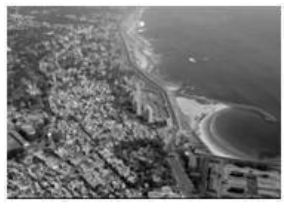

(a)

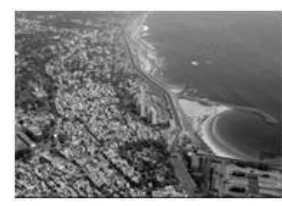

(b)

Fig 11: case-II-Vizag city Costal Region: (a) Proposed MHMF Filtered Merged Image after performing Horizontal Sub-image Filtering (MHMH) (b) Proposed combination (MHMFMOD)

TABLE 1. Performance Metrics of Different Filters Evaluated Quantitatively for Road Image of Case-II-Vizag City Costal Region with Salt \& Pepper Noise of density 0.04 (i.e. $4 \%$ of total pixels being corrupted by impulse noise)

\begin{tabular}{|c|c|c|c|c|c|c|c|c|c|c|c|c|}
\hline $\begin{array}{c}\text { formance } \\
\text { Metrics } \\
\text { Filter } \\
\text { Name }\end{array}$ & MSE & RMSE & SNR & PSNR & NCC & $A D$ & SC & MD & MAE & NAE & $\mathbf{Q}$ & EME \\
\hline AF & 269.5897 & 16.4192 & 0.0897 & 23.8238 & 0.9796 & -0.5769 & 1.0209 & 127 & 11.3489 & 0.1064 & 0.5675 & 17.9019 \\
\hline ALF & 410.7432 & 20.2668 & 0.0614 & 21.9951 & 0.9775 & -0.9731 & 1.0142 & 221 & 13.2377 & 0.1241 & 0.4580 & 20.3675 \\
\hline WF & 813.0878 & 28.5147 & 0.0619 & 19.0294 & 0.9965 & -3.8237 & 1.0859 & 208 & 20.1076 & 0.1884 & 0.2958 & 20.3128 \\
\hline MF & 170.6099 & 13.0618 & 0.0078 & 25.8108 & 0.9927 & -0.8750 & 1.0018 & 168 & 7.9583 & 0.0746 & 0.7600 & 18.1603 \\
\hline HMF & 161.8711 & 12.7229 & 0.1265 & 26.0391 & 0.9795 & 0.3752 & 1.0295 & 176 & 7.5906 & 0.0711 & 0.7837 & 17.2409 \\
\hline AMF & 402.4752 & 20.0618 & 0.0663 & 22.0834 & 0.9772 & -0.8994 & 1.0154 & 225 & 13.1690 & 0.1234 & 0.7587 & 20.0249 \\
\hline WMF & 174.3955 & 13.2059 & 0.1061 & 25.7154 & 0.9814 & 0.2875 & 1.0247 & 244 & 7.8246 & 0.0733 & 0.7869 & 18.2154 \\
\hline CWMF & 122.3395 & 11.0607 & 0.0545 & 27.2551 & 0.9892 & 0.1848 & 1.0126 & 161 & 6.2999 & 0.0590 & 0.8468 & 21.5956 \\
\hline PSMF & 135.6969 & 11.6489 & 0.0873 & 26.8051 & 0.9849 & 0.3792 & 1.0203 & 182 & 6.6009 & 0.0619 & 0.8408 & 20.8668 \\
\hline TMF & 53.2190 & 7.2951 & 0.0087 & 30.8701 & 0.9970 & 0.0183 & 1.0020 & 104 & 4.9472 & 0.0464 & 0.8806 & 24.4632 \\
\hline DBMF & 60.3450 & 7.7682 & 0.0067 & 30.3244 & 0.9970 & 0.2456 & 1.0015 & 137 & 5.1786 & 0.0485 & 0.8779 & 24.4460 \\
\hline BF & 101.4410 & 10.0718 & 0.0962 & 28.0687 & 0.9852 & 0.1415 & 1.0224 & 167 & 7.0490 & 0.0661 & 0.7291 & 18.0938 \\
\hline RMF & 168.1247 & 12.9663 & 0.1144 & 25.8745 & 0.9807 & 0.3703 & 1.0267 & 168 & 7.7674 & 0.072 & 0.7886 & 18.0657 \\
\hline FSMF & 68.0467 & 8.2490 & 0.0122 & 29.8027 & 0.9960 & 0.0396 & 1.0028 & 138 & 5.2901 & 0.0496 & 0.8728 & 23.7012 \\
\hline \multicolumn{13}{|c|}{ Proposed Method } \\
\hline MHMF & 62.9578 & 12.7655 & 0.1265 & 29.0101 & 0.9796 & 0.1550 & 1.0296 & 183 & 4.8395 & 0.0715 & 0.7837 & 17.3754 \\
\hline IMHMH & 62.7945 & 12.7591 & 0.1270 & 29.0144 & 0.9795 & 0.1315 & 1.0297 & 181 & 5.5980 & 0.0712 & 0.7847 & 17.3519 \\
\hline IMHMV & 61.5873 & 12.7117 & 0.1275 & 29.0467 & 0.9794 & 0.1218 & 1.0298 & 162 & 5.5801 & 0.0710 & 0.7850 & 17.2509 \\
\hline $\begin{array}{c}\text { MHMFM } \\
\text { OD }\end{array}$ & 53.1156 & 7.2948 & 0.1231 & 30.7170 & 0.9773 & -0.0228 & 1.0007 & 143 & 4.9350 & 0.0481 & 0.8778 & 24.1333 \\
\hline
\end{tabular}


TABLE 2: Performance Metrics of Different Filters Evaluated Quantitatively for Road Image of Case- II-Vizag City Costal region with Salt \& Pepper Noise of density 0.2 (i.e. $20 \%$ of total pixels being corrupted by impulse noise)

\begin{tabular}{|c|c|c|c|c|c|c|c|c|c|c|c|c|}
\hline $\begin{array}{c}\begin{array}{c}\text { Performance } \\
\text { Metrics }\end{array} \\
\text { Filter Name }\end{array}$ & MSE & RMSE & SNR & PSNR & NCC & $A D$ & SC & MD & MAE & NAE & $\mathbf{Q}$ & EME \\
\hline AF & 707.8310 & 26.6051 & -0.1203 & 19.6315 & 0.9874 & -3.9705 & 1.0927 & 181 & 20.3457 & 0.1907 & 0.3702 & 22.2082 \\
\hline ALF & 812.0270 & 28.4961 & -0.0705 & 19.0351 & 0.9776 & -3.8819 & 1.0983 & 229 & 20.0993 & 0.1884 & 0.2973 & 20.2227 \\
\hline WF & 720.0409 & 26.8336 & -0.0260 & 19.5572 & 0.9758 & -3.8314 & 0.9940 & 238 & 18.9845 & 0.1779 & 0.2953 & 21.9526 \\
\hline MF & 263.2793 & 16.2259 & 0.0895 & 23.9266 & 0.9799 & 0.3532 & 1.0208 & 254 & 9.3454 & 0.0876 & 0.7219 & 20.8450 \\
\hline HMF & 271.1811 & 16.4676 & 0.0956 & 23.7982 & 0.9789 & 0.3289 & 1.0222 & 234 & 9.3488 & 0.0876 & 0.6928 & 21.1136 \\
\hline AMF & 471.5683 & 21.7156 & -0.0077 & 21.3954 & 0.9831 & -0.5075 & 0.9982 & 183 & 13.0315 & 0.1221 & 0.6466 & 22.2583 \\
\hline WMF & 291.1951 & 17.0644 & 0.0740 & 23.4890 & 0.9806 & 0.1905 & 1.0172 & 243 & 9.5369 & 0.0894 & 0.7205 & 21.0515 \\
\hline CWMF & 211.5123 & 14.5435 & 0.0392 & 24.8774 & 0.9875 & 0.1633 & 1.0091 & 237 & 7.7729 & 0.0728 & 0.7779 & 22.7101 \\
\hline PSMF & 182.6275 & 13.5140 & 0.0763 & 25.5151 & 0.9844 & 0.4400 & 1.0177 & 226 & 7.1821 & 0.0673 & 0.8509 & 21.2042 \\
\hline TMF & 84.1325 & 9.1724 & 0.0277 & 28.8812 & 0.9936 & 0.0585 & 1.0064 & 170 & 5.8039 & 0.0544 & 0.8387 & 23.5309 \\
\hline DBMF & 107.8150 & 10.3810 & 0.0235 & 27.8061 & 0.9932 & 0.3129 & 1.0054 & 178 & 6.2702 & 0.0588 & 0.8525 & 24.1880 \\
\hline BF & 101.8150 & 10.0903 & 0.0974 & 28.0527 & 0.9851 & 0.1477 & 1.0227 & 175 & 9.0587 & 0.0662 & 0.7287 & 18.3359 \\
\hline RMF & 265.3835 & 16.2906 & 0.0855 & 23.8921 & 0.9802 & 0.2974 & 1.0199 & 235 & 9.2936 & 0.0871 & 0.7226 & 20.7930 \\
\hline FSMF & 139.2051 & 11.7985 & 0.0506 & 26.6943 & 0.9890 & 0.1696 & 1.0117 & 200 & 6.5891 & 0.0618 & 0.8601 & 24.0075 \\
\hline \multicolumn{13}{|c|}{ Proposed Method } \\
\hline MHMF & 106.3438 & 11.6236 & 0.0965 & 23.7163 & 0.9786 & 0.3119 & 1.0225 & 215 & 9.3789 & 0.0879 & 0.6927 & 24.7725 \\
\hline IMHMH & 107.7071 & 10.4532 & 0.0914 & 23.8058 & 0.9794 & 0.2584 & 1.0213 & 247 & 9.2656 & 0.0568 & 0.7016 & 20.3713 \\
\hline IMHMV & 108.0562 & 10.6750 & 0.0913 & 23.6895 & 0.9791 & 0.2566 & 1.0213 & 251 & 9.3531 & 0.0877 & 0.6906 & 20.5142 \\
\hline MHMFMOD & 84.9179 & 10.1940 & 0.0917 & 27.9639 & 0.9841 & -0.0308 & 1.0040 & 181 & 5.9464 & 0.0557 & 0.8605 & 23.5622 \\
\hline
\end{tabular}

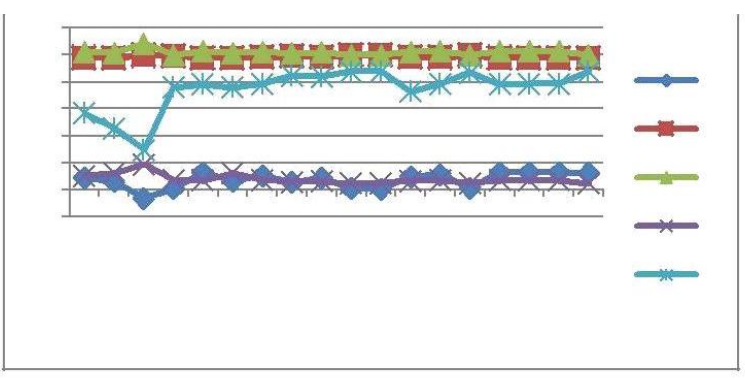

Fig. 14 Comparison of Performance of Different Filters with Noise Density 0.04 vs.

[SNR, NCC, AD, SC, NAE andQ] Objective Metrics for Satellite Image of Vizag city costal region.

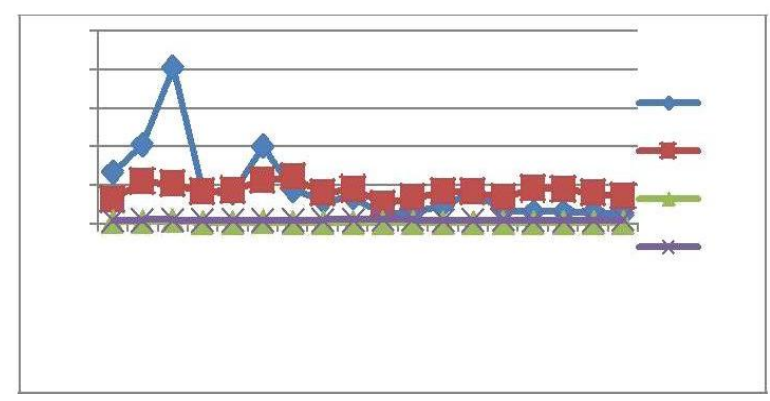

Fig. 15 Comparison of Performance of Different Filters with Noise Density 0.04 vs. [MSE, MAE, EME \& MD] Objective Metrics for Satellite Image of Vizag city costal region

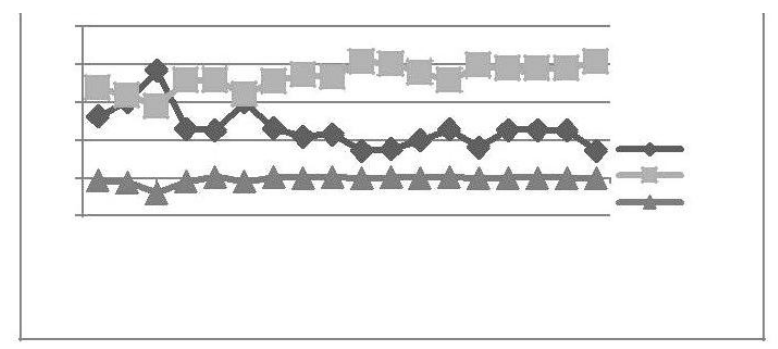

Fig. 16 Comparison of Performance Different Filters with Noise Density 0.04 vs. [RMSE, PSNR \& AD] Objective Metrics for Satellite Image of Vizag city costal region.

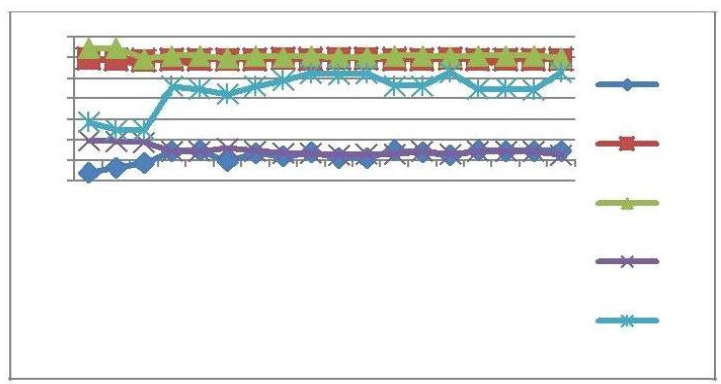

Fig. 17 Comparison of Performance of Different Filters with Noise Density 0.2 vs. [SNR, NCC, AD, SC, NAE and Q] Objective Metrics for Satellite Image of Vizag city costal region 


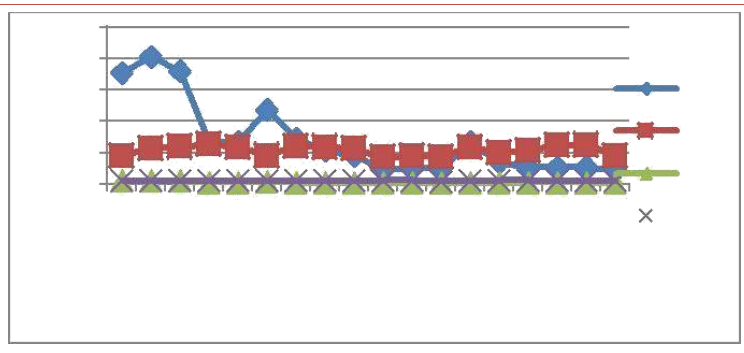

Fig. 18 Comparison of Performance of Different Filters with Noise Density 0.2 vs. [MSE, MAE, EME \& MD] Objective Metrics for Satellite Image of Vizag city costal region.

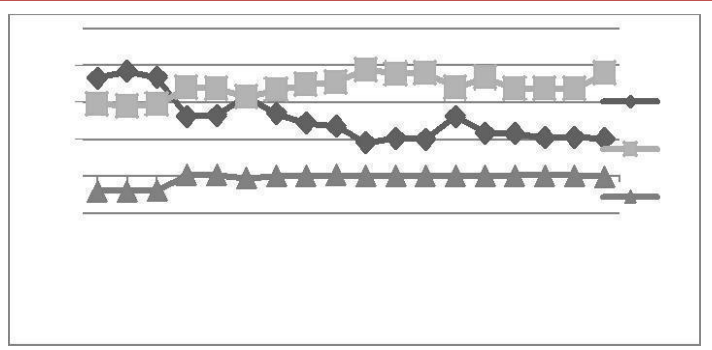

Fig. 19 Comparison of Performance Different Filters with Noise Density 0.2 vs. [RMSE, PSNR \& AD] Objective Metrics for Satellite Image of Vizag city costal region.

The quantitative results in terms of 12 objective measurements, such as, MSE, RMSE, SNR, PSNR, NCC, $A D, S C, M D, M A E, N A E, Q$ and EME have been given in Table 2 and Table 3 for the test image corrupted by impulse noise with standard deviation (SD) 0.04 and 0.2 respectively. The comparative analysis of visual observations has been presented in Fig. 2-13 for the test image. Comparison graphs were shown in Fig.1416 with impulse noise having SD 0.04 and the same have been shown in Fig.17-19 with impulse noise having SD 0.2. Note that the value tabulated in Table 2 and Table 3 of proposed filter is always maintaining better result than that of other filters. It can be seen that the SNR performance is significantly improving by the MHMFMOD filter with appropriate window size. The vertical and horizontal split sub-images scored a good result, respectively, and this is still significantly better than the few other methods. MHMF holds low MSE, RMSE and high SNR, PNSR and Q. It also gives better performance than others filters reported in this paper with respect to MD and NAE. However it is found that filter CWMF, TMF and DBMF take less execution time than MHMF based methods. The results obtained using MHMF technique ensures noise free and good quality of the image as well. Experimental results show the feasibility of the filter. This is affirmed by auditing the numerical measures like SNR, PSNR, MSE, NAE, MD, $Q$ and visual observations. In the last row of Table 2 and Table 3, we can see that our proposed combination MHMFMOD is clearly superior to all other methods in most of the cases.

\section{Conclusion}

In this paper, a Multistage Hybrid Median filtering (MHMF) method is presented to address the limitation of most median based filter. Further, we have shown that by dividing the input image into sub-images, and performing the proposed MHMF on each sub-image individually, the performance can be significantly improved. By combining two filtered sub-image representations, the overall performance can be improved even further. We tested all the described techniques on the case database images and showed that our proposed filter gives significantly better results for the test images. The preliminary results, both on standard median filtering and the modified versions, show promising results and justify further research of the MHMF for the pre-processing and illumination changes task. Furthermore, we presented and compared results for these filtering techniques. Experimental results have shown the feasibility of the new filter as well as taking into account the local features such as the possible presence of edges. This kind of approaches can be useful for Image pre-treatment of roads (or roads seeds) and help to decide the most suitable emergency transportation route in disastrous area. This can also be useful for assessing 
the extent of damages and for making decisions on disaster mitigation immediately after a massive natural calamity.

\section{REFERENCES}

[1] S. Voigt, T. Kemper, T. Riedlinger, R. Kiefl, K. Scholte, and H. Mehl, -Satellite image analysis for disaster and crisis-management support, IEEE Transactions on Geoscience and Remote Sensing, vol. 45, pp. 15201528, June 2007.

[2] S.George S. Percivall, III, Senior Member, IEEE, Nadine Alameh, Hervé Caumont, Karen L. Moe, and John D. Evans, "Improving Disaster Management Using Earth Observations-GEOSS and CEOS Activities" IEEE journal of selected topics in applied earth observations and remote sensing, vol. 6, no. 3, June 2013

[3] A.M. D. YANG, T. C. SU, C. H. HSU, K. C. CHANG and M. WU, -Mapping of the 26 December 2004 tsunami disaster by using FORMOSAT-2 images, International Journal of Remote Sensing, Vol. 28, Nos. 13-14, July 2007, 3071-3091

[4] http://www.nrsc.gov.in

[5] H. Faraji and W.J. MacLean, - CCD noise removal in digital images, IEEE Transactions on image processing, vol. 15, 2006, pp. 2676-2685

[6] Md. Abdul Alim sheikh, S. Mukhopadhyay, "Comparative Analysis of Noise Reduction Techniques for Image Enhancement ", IJIRD, PP: 103- 112, volume 2 Issue 12, December 2013.

[7] A Chambolle. et al., (1998) - Nonlinear Wavelet Image Processing: Variational Problems, Compression and Noise Removal through Wavelet Shrinkage , IEEE Trans. Image Processing, 7, pp. 319-335.

[8] J. F. Abramatic and L. M. Silverman, - Nonlinear restoration of noisy images, IEEE Trans. Pattern Anal. Mach. Intell., vol. PAMI-4, pp. 141-149, Mar. 1982.

[9] D.L. Donoho, I.M. Johnstone, (1994) -Ideal Spatial Adaptation by Wavelet Shrinkage, Biometrika, 81, No. 3, pp. 425-455.

[10] S. Mallat, (1989) -A Theory for Multiresolution Signal Decomposition: the Wavelet Representation, IEEE Trans. on Patt. Anal. Mach Intell., 11, pp. 674-693.

[11] P. Perona and J. Malik, (1990) - Scale-space and Edge Detection using Anisotropic Diffusion, IEEE Trans.Pattern Analysis and Machine Intelligence, 12, No. 7, pp. 629-639.

[12] C. Tomasi and R. Manduchi, -Bilateral Filtering forGray and Color Images, in Proc. Int. Conf. ComputerVision, pp. 839-846, 1998.

[13] D. L. Donoho, (1995) -De-noising by Soft Thresholding, IEEE Trans. on Inform, Theory, 41, No. 3, pp. 613- 627.

[14] S G Chang, B. Yu, and M. Vetterli,-Adaptive Wavelet Thresholding for Image Denoising and Compression, IEEE Transactions on Image Processing, vol. 9, no. 9, pp. 1532 -1546, September 2000. 
K.Kiran Reddy, P.Bhsskara Redd and P.Amarender Reddy; Associate-Image Filtering Method with Enhanced Denoising Feature for Road Detection in Disaster Management. Transactions on Machine Learning and Artificial Intelligence, Volume 4 No 6 December (2016); pp: 50-63

[15] N.C.Gallagher,Jr. and G.L.Wise, -A Theoretical Analysis of the Properties of Median Filters, IEEE Trans. Acoust., Speech and Signal Processing, vol.ASSP-29, pp.1136-1141, Dec. 1981.

[16] D. R. K. Brownrigg,-The weighted median filter, Commun. ACM, vol. 27, no. 8, pp. 807-818, Aug. 1984.

[17] A. Nieminen, P. Heinonen, and Y. Neuvo, -A new class of detail preserving filters for image processing, IEEE Trans. Pattern Anal.Mach. Intell., vol. PAMI-9, pp. 74-90, Jan. 1987.

[18] P. Heinonen and Y. Neuvo, -FIR-median hybrid filters, IEEE Trans. Acoust., Speech, Signal Processing, vol. ASSP-35, pp. 832-838, June 1987.

[19] Zhang, S., and Karim, M.A.: _A new impulse detector for switching median filters', IEEE Signal Process. Lett. 2002, 9, (11), pp. 360-363.

[20] H Hwang and R A Haddad, -Adaptive Median Filter: New Algorithms and Results, IEEE Transactions on image processing Vol 4 No 4 April 1995

[21] H.-M. Lin and A. N. Willson, Jr., -Median filters with adaptive length, IEEE Trans. Circuits Syst., vol. CAS35. pp. 675-690, June 1988.

[22] R. Ding and A. N. Venetsanopoulos, - Generalized homomorphic and adaptive order statistic filters for the removal of impulsive and signal-dependent noise, IEEE Trans. Circuits Syst ., vol. CAS-34, pp. 948955, Aug. 1987.

[23] L. Lin , R. Yang, M. Gabbouj and Y. Neuvo, -Weighted Median Filters: A Tutorial, IEEE Transactions On Circuits And Systems-II: Analog And Digital Signal Processing, VOL. 43, NO. 3, MARCH 1996.

[24] Sung-Jea Ko and Y. H. Lee, -Centre Weighted Median Filters and Their Applications to image Enhancement, IEEE Transactions on Circuits and Systems, Vol. 38, No. 9, September 1991.

[25] C. L. Mallows, -Some theory of non-linear smoothers, The Annals of Statistics, vol. 8, pp. 695-715, 1980.

[26] Zhou Wang and David Zhang, - Progressive Switching Median Filter for the Removal of Impulse noise from Highly Corrupted Images, IEEE Transaction on circuits and systems-II: analog and digital signal processing Vol. 46 No 1 January 1999.

[27] T. Chen, K.-K. Ma, and L.-H. Chen, -Tri-state median filter for image denoising, IEEE Transactions on Image Processing, vol. 8, no. 12, pp. 1834-1838, 1999.

[28] D. A. F. Florencio and R. W. Schafer, -Decision-based median filter using local signal statistics, in Visual Communications and Image Processing '94, vol. 2308 of Proceedings of SPIE, pp. 268-275, Chicago, III, USA, September 1994.

[29] Abdessamad ben hamza, Pedro I luque-escamilla, Jos'e mart'inez-aroza, and Ramon roman-roldan, -Removing Noise and Preserving Details with Relaxed Median Filters, Journal of Mathematical Imaging and Vision 11, 161-177 (1999) Kluwer Academic Publishers. Manufactured in the Netherlands. 
[30] G. R. Arce and R. E. Foster, -Detail preserving ranked-order based filters for image processing, IEEE Trans. Acoust .. Speech,Signal Processing, vol. 37, pp. 83-98, Jan. 1989.

[31] Zhang, D.S.; Kouri, OJ. - Varying weight trimmed mean filter for the restoration of impulse noise corrupted images, Acoustics, Speech, and Signal Processing IEEE International Conference. vol. 4. pp. 137-140. 2005.

[32] J. S. Lee, -Digital image enhancement and noise filtering by use of local statistics, IEEE Trans. Pattern Anal. Mach. Intell., vol. PAMI-2, pp. 165-168, Mar. 1980.

[33] Sun, T., and Neuvo, Y.: = Detail-preserving median based filters in image processing', Pattern Recognit. Lett., 1994, 15, (4), pp. 341-347.

[34] J. Najeer Ahamed and V. Rajamani, —Design of Hybrid Filter for Denoising Images Using Fuzzy Network and Edge Detecting, American Journal of Scientific Research ISSN 1450-223X Issue 3(2009), pp.5-14.

[35] F. Russo and G. Ramponi, -A fuzzy operator for the enhancement of blurred and noisy images, IEEE Trans. Image Processing, vol. 4, pp. 1169-1174, Aug. 1995.

[36] C.-S. Lee, Y.-H. Kuo, and P.-T. Yu, - Weighted fuzzy mean filters for image processing, Fuzzy Sets Syst., no. 89, pp. 157-180, 1997.

[37] C.S. Lee, Y.H. Kuo, Adaptive fuzzy filter and its application to image enhancement, in: E.E. Kerre, M. Nachtegael (Eds.), Fuzzy Techniques in Image Processing, Springer, New York, 2000, pp. 172-193.

[38] J.H. Wang, W.J. Liu, L.D. Lin, -An histogram-based fuzzy filter for image restoration, IEEE Trans. Systems Man and Cybernetics Part B Cybernetics 32 (2) (2002) 230-238.

[39] H. Xu, G. Zhu, H. Peng, D.Wang, Adaptive fuzzy switching filter for images corrupted by impulse noise, Pattern Recognition Lett. 25 (2004)1657-1663.

[40] Dimitri Van De Ville, Mike Nachtegael, Dietrich Van der Weken, Etienne E. Kerre, Wilfried Philips, IEEE, and Ignace Lemahieu - Noise Reduction by Fuzzy Image Filtering, IEEE TRANSACTIONS ON FUZZY SYSTEMS, VOL. 11, NO. 4, AUGUST 2003.

[41] Ahmet M. Eskicioglu, Paul S. Fisher, - Image Quality Measures and Their Performance IEEE Transactions on Communication, Vol. 43, No. 12, pp. 2959-2965, December 1995.

[42] Zhou Wang, Alan C. Bovik, -A Universal Image Quality Index, IEEE SIGNAL PROCESSING LETTERS, VOL. 9, NO. 3, MARCH 2002.

[43] K. Panetta,Y. Zhou, S. A Gaian and H. Jia. -Nonlinear Unsharp Masking for Mammogram Enhancement . IEEE Transactions on Information Technology in Biomedicine, 2011, 15(6): 918-928. 